\title{
503 - Informal antidepressant strategies in nursing homes: Two Group Concept Mapping studies among residents, their relatives, and professional caregivers Inge Knippenberg - Ruslan Leontjevas - Slavi Stoyanov - Anke Persoon - Peter Verboon - Hester Vermeulen - Jacques van Lankveld - Debby Gerritsen
}

Background: Although formal treatments like antidepressants and psychotherapy may effectively reduce depression in nursing home residents, side effects and poor treatment adherence are common. To improve depression care, it is important to also learn from informal strategies already used successfully in daily practice, alongside or in absence of formal treatment. For example, although not prescribed as formal treatment, a care provider may seat a resident with depression at a table near the window. This may have antidepressant effects due to extra day light or pleasant views from the window.

Objectives: To identify, categorize, and prioritize informal antidepressant strategies for residents already used in daily practice as reported by residents themselves, their relatives, and professional caregivers.

Method: In the first Group Concept Mapping study, residents, relatives, and professional caregivers $(N=$ 124) brainstormed on strategies to prevent or improve depression that may be performed by residents themselves. In the second study, the same participants $(N=110)$ reported on strategies others involved in residents' lives could perform. In a second round of both studies, participants rated the expected effectiveness ( $N=54, N=51$ respectively) and feasibility ( $N=50$ for both studies) of the strategies mentioned. In addition, strategies for both studies were sorted based on similarity in meaning by experts.

Results: Six clusters appeared for actions to be undertaken by residents themselves, and five clusters for actions by others. The results showed that, for strategies by residents, the clusters 'Being socially connected' and 'Participating in activities', and for strategies by others, the cluster 'Offering personal

attention' stand out the most in terms of high expected effectiveness. The cluster with strategies executed by residents that stood out as the most feasible was 'Having a healthy living environment'. The most feasible clusters performed by others were 'Offering personal attention', 'Using positive treatment/approach', and 'Using or adapting the physical environment'.

Conclusion: By using an innovative bottom-up participatory approach, this research shed light on various clusters of useful informal antidepressant strategies for daily practice. To test effectiveness and implementation, research is needed on these antidepressant strategies and their regular use in care.

\section{4 - Periodontal Disease and Risk of Dementia in Medicare Patients with Hepatitis C Virus Joseph E. Malone, Linh Tran, Jeah Jung, Chen Zhao}

Objective: To examine whether periodontal disease increases the risk of developing Alzheimer's disease and related dementias (ADRD) among hepatitis $C$ patients in Medicare claims data.

Background: Periodontal disease and hepatitis C virus (HCV) represent chronic infectious statesthat are common in elderly adults. Both conditions have independently been associated with an increased risk for dementia. Chronic infections are thought to lead to neurodegenerative changes in the central nervous system possibly by promoting a proinflammatory state. This is consistent with growing literature on the etiological role of infections in dementia. No studies have evaluated the association of periodontal disease with dementia in HCV patients. 